\title{
Quantification of the flame structure at multi-scale levels
}

\author{
G. Tan (谭贵文), ${ }^{1}$ U. Ahmed, ${ }^{2}$ L. Wang (王利坡), ${ }^{1,}$ a) and N. Chakraborty ${ }^{2}$ \\ ${ }^{1)}$ UM-SJTU Joint Institute, Shanghai Jiao Tong University, Shanghai 200240, China \\ ${ }^{2)}$ School of Engineering, Newcastle University, Claremont Road, Newcastle-Upon-Tyne, NE1 7RU, \\ $U K$
}

(Dated: 18 November 2020)

The interaction between flame and turbulence leads to the complex flame topology at multiple scales. In this work the non-local statistical features of the flame surface topology in turbulent premixed combustion are investigated by using dissipation element (DE) analysis. Considering a spatial point on the flame front, the gradient trajectory of the flame curvature reaches a local maximum curvature point and a local minimum curvature point along the ascending and descending directions, respectively. A dissipation element (DE) is the patch consisting of the points whose gradient trajectories share the same pair of extremal points. The characteristic parameters are chosen as the distance and the curvature difference between two extremal points of each DE. Multi-scale DEs can also be defined to improve the understanding of the turbulent premixed flame surface topology at various scales by extending the extremal points at different scale levels. Results show that the turbulence intensity determines the flame corrugation and consequently the number of DEs, whereas the probability density functions (PDF) of the normalized length scale of DEs remain unchanged. The conditional mean of the curvature difference with respect to the length scale is strongly dependent on the influence of turbulence on the flame, i.e. that scaling slope decreases with increasing turbulence intensity. However, for a given turbulence intensity, the scaling slopes of such conditional mean remain unchanged in different scale brackets.

\section{INTRODUCTION}

In premixed turbulent combustion the interaction between the flame and turbulence leads to the fluctuation of scalars, e.g. species and temperature fields, at multiple scales. The flame surface inherits the imprint of such interaction and consequently exhibits corrugation involving multiple scales. Quantitative characterization of the flame topology is important to understand the kinematics and dynamics of turbulent combustion. For instance, the premixed flame curvature and the tangential strain rate are related to each other, which in turn influence the flame propagation statistics ${ }^{1}$. Under the flamelet assumption, such relation is crucial for the $G$-equation and the Flame Surface Density (FSD) based closure methodologies $^{1,2}$.

From the statistical point of view, curvature and strain rate are pointwise and local properties. In a given turbulent flow field, it is also important to understand the non-local features with respect to the spatial scales. For instance, conditional structure functions introduced by Sabelnikov et al. ${ }^{3,4}$ and explored by Brearley et al. ${ }^{5}$ to analyze the non-local features with respect to the spatial scales in terms of the fluid velocity and the impact of thermal expansion induced by the flame has on the velocity distribution. The main challenge of nonlocal statistics related to flame topology lies in the quantitative, rather than illustrative, structure identification, which is relatively rare in the existing literature. A novel attempt in this regard is the dissipation element (DE) analysis ${ }^{7}$. In a flow field, each spatial point is associated with a trajectory along which the scalar value changes monotonically along the local

\footnotetext{
${ }^{a)}$ Electronic mail: Lipo.Wang@sjtu.edu.cn
}

scalar gradient direction between a pair of local maximal and minimal points, where the scalar gradient vanishes. Thus, the entire flow field can be partitioned into space-filling dissipation elements, each of which consists of trajectories sharing the same pair of local extremal points. DE analysis presents a possibility for feature tracking of space-filling structures, which can be beneficial from the point of view of the development of reduced order models, as to be discussed hereafter.

Physically, local extremal points and thus the scalar gradient trajectories are determined by the perturbation from turbulent eddies. In accordance to the multi-scale property of turbulence, such perturbation exists at different scales. In studying the one-dimensional stochastic process, a so-called multi-level segment method introduces the extremal points at different levels by considering extremum inside a preset window size ${ }^{10}$. It was found that the scaling range of an equivalently defined structure function can be largely extended, and the scaling relation fits better with the dimensional argument prediction. A similar idea can be extended to define DEs at different scale levels. The advantages of such extension can be summarized at least from the following two aspects. First, the structures identified can be extended from the fine scale level to large scale level to cover a broader spectrum range; second, the effect of data noises on structure definition can be effectively removed ${ }^{10}$.

In the present work, the flame curvature $\kappa$ is chosen as the featured quantity for structure identification. According to the definition $\kappa=-0.5 \nabla \cdot(\nabla c /|\nabla c|)$, where $c$ is the progress variable defined in terms of the normalized fuel mass fraction, positive (negative) $\kappa$ implies that the flame surface is convex (concave) towards the reactants. Under the action of turbulent eddies at different sizes, the flame corrugates differently. The general idea is to investigate statistical properties of DEs defined from the flame curvature. In order to do so, a three- 
dimensional direct numerical simulation (DNS) database of statistically planar premixed flames subjected to forced unburned gas turbulence has been considered.

The paper is organized as follows. In the next section the details of the direct numerical simulation (DNS) data are provided. This is followed by the mathematical description of the multi-level dissipation element structure algorithm used in this work. The results are presented and discussed in section IV. Finally the conclusions are drawn in the last section.

\section{DIRECT NUMERICAL SIMULATION DATA}

The three-dimensional premixed turbulent reacting flow is numerically simulated using a well-known code SENGA $+{ }^{11}$.The numerical implementation of the spatial derivative adopts a 10th order central difference scheme for the internal grid points while the order of accuracy gradually decreases to one-sided 2nd order scheme at the non-periodic boundary points. The temporal integration is based on an explicit third-order low storage Runge-Kutta scheme $^{12}$. The computational domain has dimensions of $L_{x_{1}}=140.5 \delta_{Z}, L_{x_{2}}=L_{x_{3}}=70.18 \delta_{Z}$, where $\delta_{Z}=\alpha_{T} / S_{L}$ is the Zel'dovich flame thickness with $\alpha_{T}$ and $S_{L}$ refering to the thermal diffusivity of unburned mixture and unstrained laminar flame speed, respectively. A uniform Cartesian mesh with $800 \times 400 \times 400$ is used, which ensures about 10 grid points across the thermal flame thichness $\delta_{t h}$, defined as $\delta_{t h}=\left(T_{a d}-T_{R}\right) / \max |\nabla T|_{L}$, where $T, T_{a d}$ and $T_{R}$ are the instantaneous temperature, the adiabatic flame temperature and the reactant gas temperature, respectively. In the present analysis, all the thermo-physical properties (i.e. viscosity, thermal conductivity and density-weighted mass diffusivity) are taken to be constant and independent of temperature for the purpose of simplicity. Thus, $\delta_{t h} / \delta_{Z}$ is found to be 1.77 for the database considered here. The grid spacing also ensures 1.5 grid points within the Kolmogorov length scale. A single-step chemical mechanism has been used for the purpose of computational economy for a detailed parametric analysis ${ }^{13,14}$ (only a small subset of all the cases is considered for this analysis and a monotonic trend in terms of turbulence intensity has been obtained for the other cases in this database). Moreover, the flame surface topology remains unaffected by the choice of chemical mechanism, and the reaction progress variable $c$ can be uniquely defined without any uncertainty in the presence of a simple single-step chemical mechanism. The Lewis number of all the species is taken to be unity and the gaseous mixture is considered to obey the ideal gas law. Standard values are taken for Prandtl number (i.e. $\operatorname{Pr}=0.7$ ), Zel'dovich number (i.e. $\beta=T_{a c}\left(T_{a d}-T_{R}\right) / T_{a d}^{2}=6.0$ with $T_{a c}$ being the activation temperature) and the ratio of specific heats (i.e. $\gamma=1.4$ ).

The simulations have been conducted in an inlet-outlet configuration where the inlet and outlet boundaries are specified in the direction of mean flame propagation. The transverse boundaries have been taken to be periodic. The outflow boundary has been considered to be partially non-reflecting and specified according to the Navier-Stokes Characteristic
TABLE I. The attributes of the DNS database considered for this analysis.

\begin{tabular}{lcccccl}
\hline Cases & $R e_{t}$ & $u^{\prime} / s_{L}$ & $l_{t} / \delta_{t h}$ & $D a$ & $K a$ & Regime \\
\hline A & 44.5 & 2.5 & 3.0 & 1.2 & 2.28 zones \\
\hline B & 89.0 & 5.0 & 3.0 & 0.6 & 6.5 & zones \\
\hline C & 133.5 & 7.5 & 3.0 & 0.3 & 18.3 zones \\
\hline
\end{tabular}

Boundary Conditions (NSCBC) technique ${ }^{15}$. The mean inlet velocity $U_{\text {mean }}$ has been gradually altered to match the turbulent flame speed so that the flame remains stationary in the statistical sense within the computational domain. A recently proposed modified bandwidth filtered forcing method ${ }^{16}$ in physical space has been used in the unburned gas ahead of the flame, which not only maintains the prescribed turbulence intensity $u^{\prime} / S_{L}$ but also provides the required integral length scale to flame thickness ratio $l_{t} / \delta_{t h}$, where $l_{t}$ is calculated based on turbulent kinetic energy $k$ and dissipation rate $\varepsilon$ evaluated over the whole domain as $l_{t}=(2 \mathrm{k} / 3)^{1.5} / \varepsilon$.

The inlet values of root-mean-square turbulent velocity fluctuation normalised by the unstrained laminar burning velocity $u^{\prime} / S_{L}$, integral length scale to thermal flame thickness ratio $l_{t} / \delta_{t h}$, Damköhler number $D a=l_{t} S_{L} / u^{\prime} \delta_{t h}$, Karlovitz number $K a=\left(u^{\prime} / S_{L}\right)^{3 / 2}\left(l_{t} / \delta_{t h}\right)^{-1 / 2}$ and $R e_{t}=\rho_{R} k^{2} / \varepsilon \mu_{R}$ are listed in Table 1 , where $\rho_{R}$ and $\mu_{R}$ are the unburned gas density and viscosity, respectively. The heat release parameter $\tau=\left(T_{a d}-T_{R}\right) / T_{R}$ is taken to be 4.5 for all the cases considered in this work. All the simulations listed in Table 1 have been continued until the turbulent kinetic energy and integral length scale attain the desired values and also the turbulent flame speed $S_{T}$ and flame surface area $A_{T}$ settle to statistically stationary values. The total simulation time remains greater than the throughpass time $t_{\text {sim }}>L_{x} / U_{\text {mean }}$ and at least 10 eddy turn over times (i.e. $t_{\text {sim }}>10 l_{t} / u^{\prime}$ ) for all cases.

The flame front is defined based on the progress variable $c=0.8$ isosurface because the maximum reaction rate of the progress variable takes place close to $c=0.8$ for the present thermo-chemistry. The same approach has been adopted in several previous analyses ${ }^{17-23}$. It is worth noting that the statistical results presented in the following will remain at least qualitatively unchanged for isosurfaces with different $c$ values. It has been demonstrated in previous studies ${ }^{18,19}$ that the curvature PDFs do not change significantly based on the choice of isosurfaces. For the sake of brevity, we focus on the discussion of the $c=0.8$ isosurface. Figure 1 shows instantaneous distributions of $c$ in the central mid-plane for the three cases. With the increase of turbulence intensity, the flame becomes increasingly corrugated. It can be expected that the DEs statistics extracted from the flame curvature will accordingly be different, which will be discussed in the next section. 


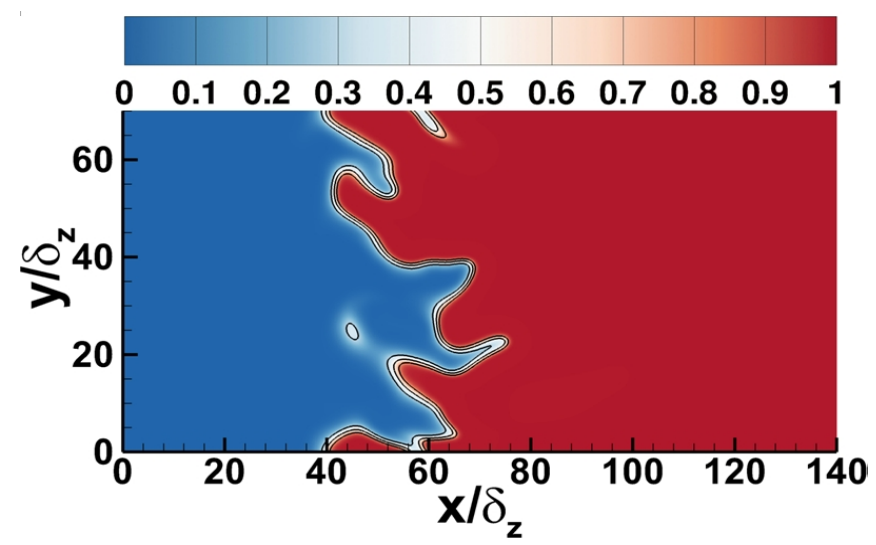

(a)

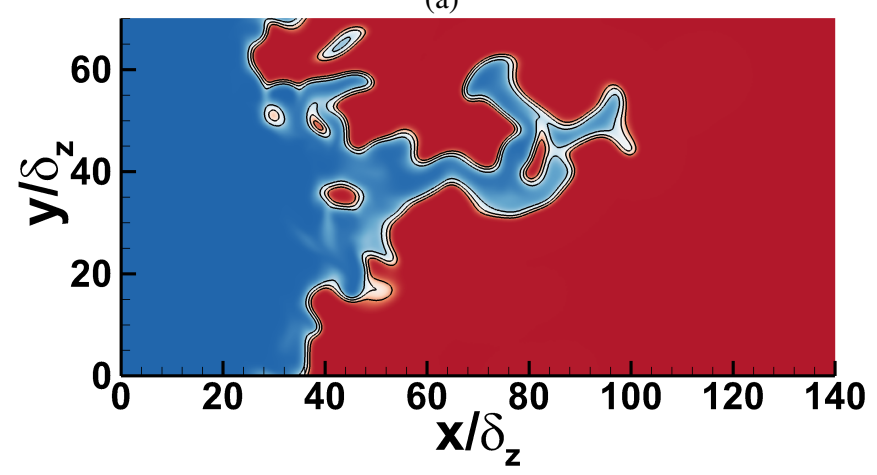

(b)

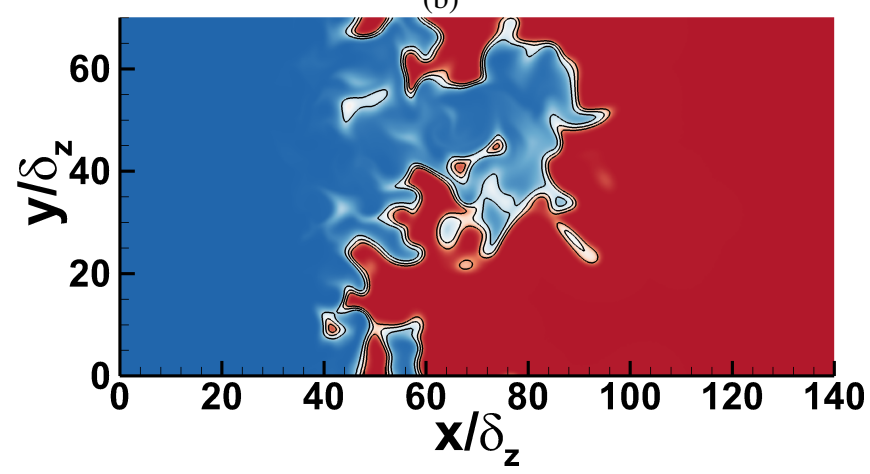

(c)

FIG. 1. Instantaneous distributions of $c$ in the central mid-plane for cases (a) A, (b) B and (c) C. The isolines of $c=0.3,0.5$ and 0.8 are shown by black contour lines from left to right.

\section{MULTI-LEVEL DISSIPATION ELEMENT STRUCTURE}

The definition of DEs at different scales is inspired from the multi-level segment idea developed by Wang \& Huang ${ }^{10}$. The framework is built upon a simple fact that extremal points are conditionally valid, with respect to a preset observation window $R$ in the following sense. The implication that a point $x_{0}$ is extremal at the $R$ scale level is

$$
\left\{\begin{array}{l}
f\left(x_{0}\right) \geq f(x), \forall x \in\left|x-x_{0}\right| \leq R, \text { (maximum) } \\
f\left(x_{0}\right) \leq f(x), \forall x \in\left|x-x_{0}\right| \leq R, \text { (minmum) }
\end{array}\right.
$$

The distance measure $|\cdot|$ can be tailored according to the questions under consideration, e.g. the typically used Euclidean distance. In this sense, the local extremal (maximum or minimum) points are valid at the scale level $R \rightarrow 0$, or $R=0^{+}$. Therefore, extremal points are conditionally valid with respect to different scale $R$, e.g. the conventional local extremal points (at the $0^{+}$level) need not still to be extremal at the $R>0$ level.

As demonstrated in one-dimensional space ${ }^{10}$, for each given scale $R$, the set of corresponding extremal points can be extracted. A one-dimensional segment at the $R$ level is defined as the part between two consecutive extremal points (at the $R$ level). Typically, the segments become larger with an increase in $R$. Thus, structures at different observation levels (i.e. different $R$ ) can be constructed. The multi-level segment approach based on this principle proves to be effective to separate the mixed statistics at different scales ${ }^{10}$. Such algorithm can be directly extended to higher dimensional cases. Generally, starting from any spatial point $P$ in a scalar field $f(\vec{x})$, the scalar gradient trajectory connects its local minimum point $P_{\min , 1}$ and local maximum point $P_{\max , 1}$. For a specified scale $R$, the trajectory along the descending direction stops at $P_{\min , 1}$, if $P_{\min , 1}$ is still the minimum point at the $R$ level. However, if $P_{\max , 1}$ is not the maximum point at the $R$ level, it implies that there is another maximum point $P_{\max , 2}$ satisfying $f\left(P_{\max , 2}\right)>f\left(P_{\max , 1}\right)$ within the spherical domain centered at $P_{\max , 1}$ with a radius $R$ (for defining the spatial distance in three-dimensional space). Then the trajectory needs to jump to $P_{\max , 2}$. Such marching process stops after $n-1$ steps till $P_{\max , n}$, a maximum point defined at the $R$ level. It is worth noting that in the above discussion $P$ is taken to be the starting point to determine trajectory. The distance between $\mathrm{P}$ and the intermediate extremal point, e.g. $P_{\max 2}$, need not to be smaller than $R$ (actually likely to be larger than $R$ ). One needs to ensure that each jump, e.g. from $P_{\max 1}$ to $P_{\max 2}$, is smaller than $R$. Therefore, at the $R$ level the gradient trajectory of point $P$ now connects $P_{\min , 1}$ and $P_{\max , n}$. The gradient trajectory between $P_{\min , 1}$ and $P_{\max , 1}$ is the case for $R=0^{+}$. When $R$ scans from 0 to some large enough quantity, DEs at multiple levels can be determined.

\section{RESULTS AND ANALYSIS}

In the present analysis, DEs are defined on the curved flame surfaces based on the extremal points of the flame surface curvature $\kappa$. An illustrative result is shown in Fig. 2. The flame surface colored with the value of $\kappa$ separates the reactant side and the product side. On the flame surface patches bounded by solid yellow lines present various DEs at the $R=0^{+}$level, each of which has one minimum (small blue dot) and one maximum point (small red dot). All of these $R=0^{+}$level DEs (totally five in Fig 2) belong to a DE at a larger $R$ level (about $5 \%$ of the domain size), whose minimum point and maximum point are presented by the large blue dot and large red dot, respectively. Since the gradient trajectory is uniquely determined, DEs at any given $R$ level are space-filling, which implies that on average the size of DEs increases with $R$, as demonstrated in Fig. 2. 


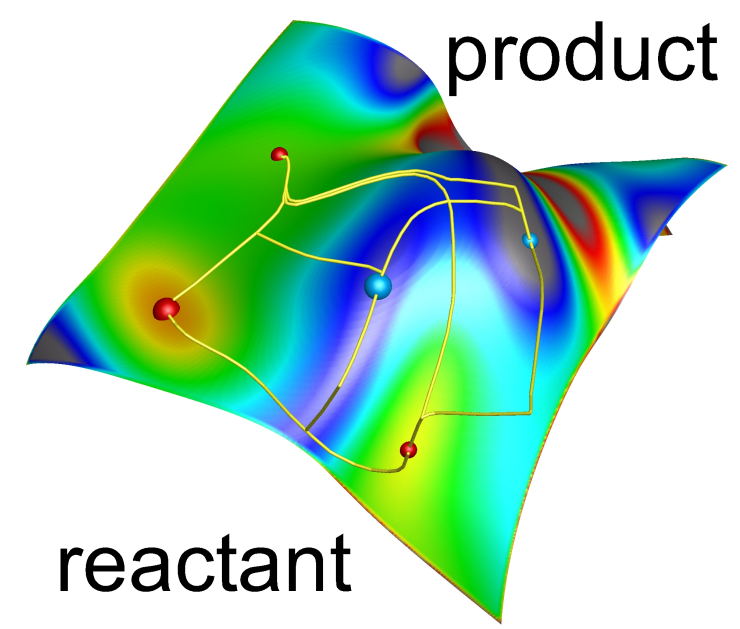

FIG. 2. Illustration of DES on the flame front colored by the local curvature, which separates the reactant and the product sides. The patches bounded by solid yellow lines represent DEs at fine scale level $\left(R=0^{+}\right)$, whose extremal points are shown in blue (for minimum) or red (for maximum) dots. These patches belong to a DE at larger $R$ level, which is about $5 \%$ of the domain size.

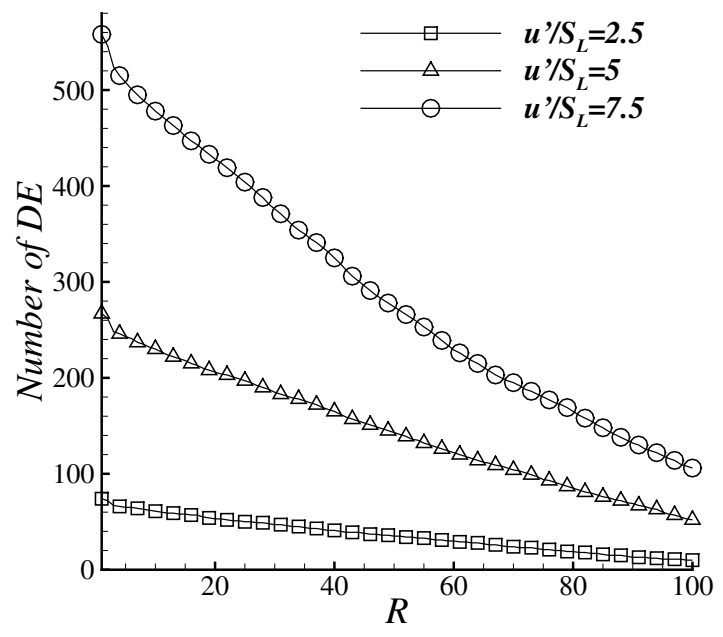

FIG. 3. Dependence of the number of DEs on the scale level $R$.

When the flame surface reaches the statistically stationary state, the DEs at different $R$ levels are identified and the change of DE number with respect to $R$ can be quantified. The results for the three cases are compared in Fig. 3. It can be seen that the DE number decreases monotonously with increasing $R$. In accordance with the definition of $\mathrm{DE}$ and the flame surface topology as shown in Fig. 1, the number of local extremal points of the flame surface curvature increases with increasing extent of flame surface corrugation. Thus, the number of DEs increases with decrease in the size of DE from case A to case $\mathrm{C}$ at $R=0^{+}$.

For each DE, the two extremal points determine $l$, the distance in between, and $\Delta \Phi=\kappa_{\max }-\kappa_{\min }$, the curvature difference in between, which are chosen as the characteristic parameters. Since $l$ changes at different $R$, it is meaningful to

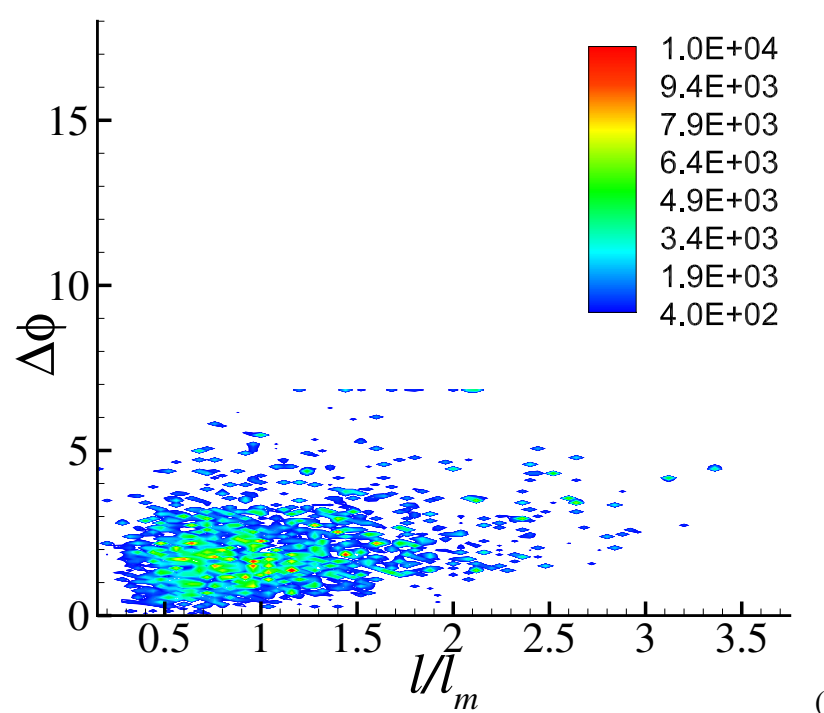

(a)

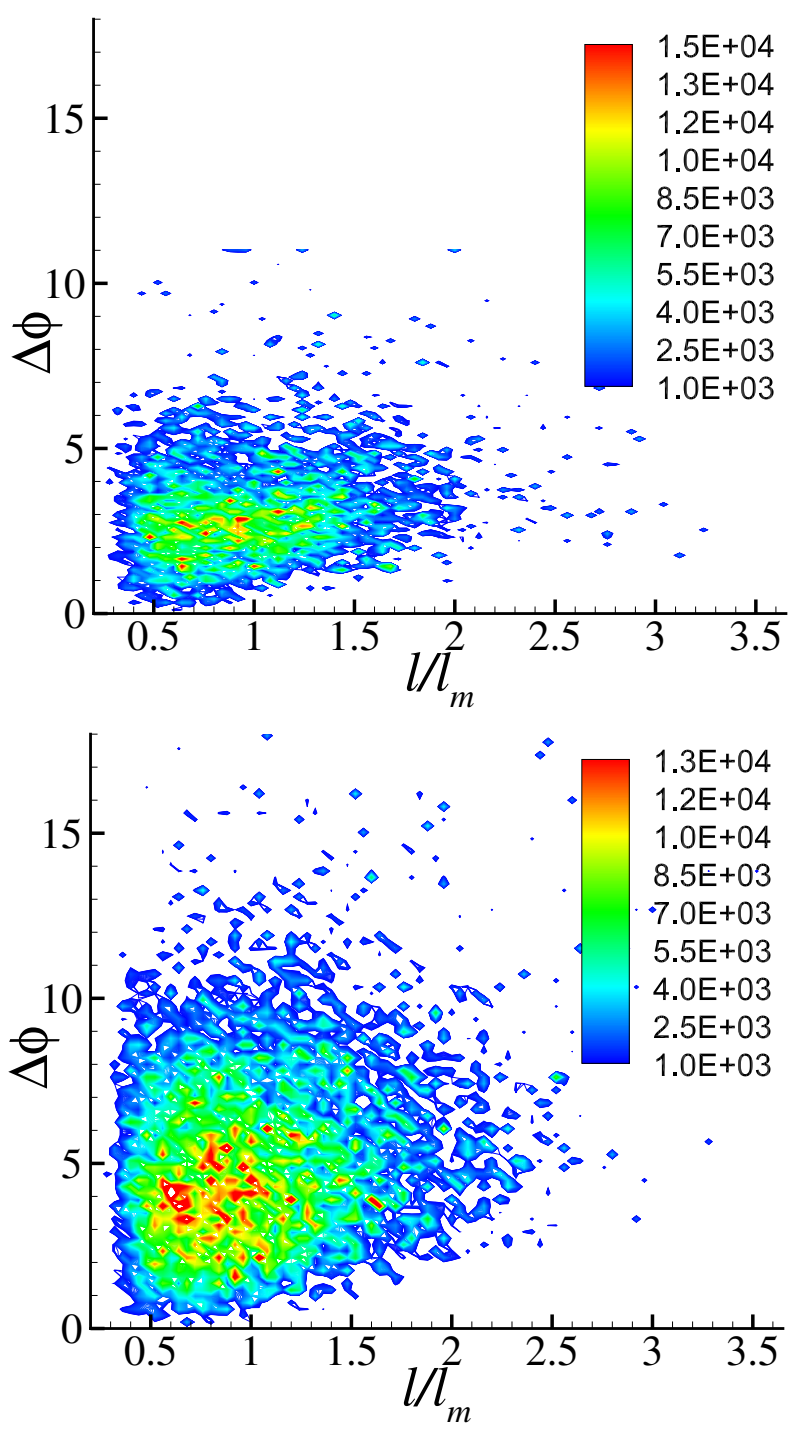

(b)

(c)

FIG. 4. (a) Joint PDFs between $\tilde{l}$ and $\Delta \Phi$ for case (a) A, (b) B and (c) C. 
consider $l$ normalized by its mean $l_{m}$, i.e. $\tilde{l}=l / l_{m}$. From a given reactive turbulence field, let $R=0^{+}$increase continuously to a large enough quantity to extract the flame structure at different scales. All the DEs obtained are cumulatively sampled to calculate the joint PDF between $\tilde{l}=l / l_{m}$ and $\Delta \kappa$. The results for all the three cases are presented in Fig. 4 and it can be noticed that the DEs are likely to appear at some finite $\tilde{l}$ and $\Delta \Phi$. Because of the considerable difference of the flame corrugation from case A to case $\mathrm{C}$, the most probable value of $\Delta \phi$ increases largely from Fig. 4 (a) to 4(c). However, the three joint PDFs remain qualitatively similar.

In order to gain a more detailed understanding of DEs at different $R$, it is helpful to know the statistics in different $R$ brackets. Figure. 5 presents the PDFs of $\tilde{l}$ in several representative $R$ ranges, including $R \in[\Delta x, 14 \Delta x], R \in[31 \Delta x, 44 \Delta x]$, $R \in[59 \Delta x, 72 \Delta x], R \in[87 \Delta x, 100 \Delta x]$, where $\Delta x=0.1 \delta_{t h}$ is the mesh size. As discussed before, the value of $R$ determines the scale level of the extracted structure, e.g., the fine scale DE structure at $R \in[1 \Delta x, 14 \Delta x]$ and the very large DE structure at $R \in[87 \Delta x, 100 \Delta x]$. Although the size of DEs changes significantly for different values of $R$, the PDFs of $\tilde{l}$ overlap. Similar results exist in the logarithmic scale as well, as shown in Fig. 6. This implies structure similarity at different scales.

In a general scenario, considering the length scale $l$ evolution in turbulence, Wang \& Peters ${ }^{7}$ proposed the following model equation for $P(\tilde{l}, t)$, the PDF of $\tilde{l}=l / l_{m}$, i.e. the length scale normalized by its mean:

$$
\begin{aligned}
& \frac{\partial P(\tilde{l}, \tilde{t})}{\partial \tilde{t}}+\frac{\partial}{\partial \tilde{l}}(\tilde{v}(\tilde{l}) P(\tilde{l}, t))=\Lambda\left[\int_{0}^{\infty} 2 P(\tilde{l}+\tilde{z}, t) d \tilde{z}-\tilde{l} P(\tilde{l}, t)\right]+ \\
& \left.8 \frac{\partial P(\tilde{l}, t)}{\partial \tilde{l}}\right|_{\tilde{l}=0}\left[\int_{0}^{\tilde{l}} \tilde{z} \tilde{\tilde{l}} P(\tilde{l}-\tilde{z}, t) P(\tilde{z}, t) d \tilde{z}-P(\tilde{l}, t)\right] .
\end{aligned}
$$

In the above equation, $\tilde{v}(\tilde{l})$ is the drift velocity at scale $\tilde{l}$, which is determined by the relative motion of two ending (extremal) points of the segment, the normalized time $\tilde{t}$ is $t D / l_{m}^{2}$ where $D$ is the reaction progress variable diffusivity, and $\Lambda$ is an eigenvalue which can be determined from the normalization condition of the PDF, i.e. $\int_{0}^{\infty} P(\tilde{l}) d \tilde{l}=1$. Physically, the evolution of $l$ is a resultant outcome from different effects. Firstly, molecular diffusion annihilates the closely located extremal points (local cusps) to smooth out the flame front, i.e. the smoothing mechanism. Secondly, turbulent random motion disturbs the flame to generate new extremal points of $\kappa$, i.e. the random perturbation mechanism. Besides these abrupt effects, the drift velocity $\tilde{v}(\tilde{l})$ describes the continuous change of $\tilde{l}$. At the statistically stationary state, there is a dynamic balance under the action of all these mechanisms and a unique time-independent solution exists for Eq. (2) ${ }^{7}$. Such counteracting mechanisms also exist for the premixed turbulent flame, i.e. perturbation from turbulent eddies of different sizes to generate wrinkles with different $\kappa$ values and the curvature dependence of local flame displacement speed ${ }^{24}$ acts to smooth the flame surface. Both Fig. 5 and Fig. 6 indicate that the solution from Eq. (2) can capture the qualitative trend of the numerical results. Especially Eq. (2) predicates an exponential decay at large $\tilde{l}^{7}$, which is in qualitative agreement
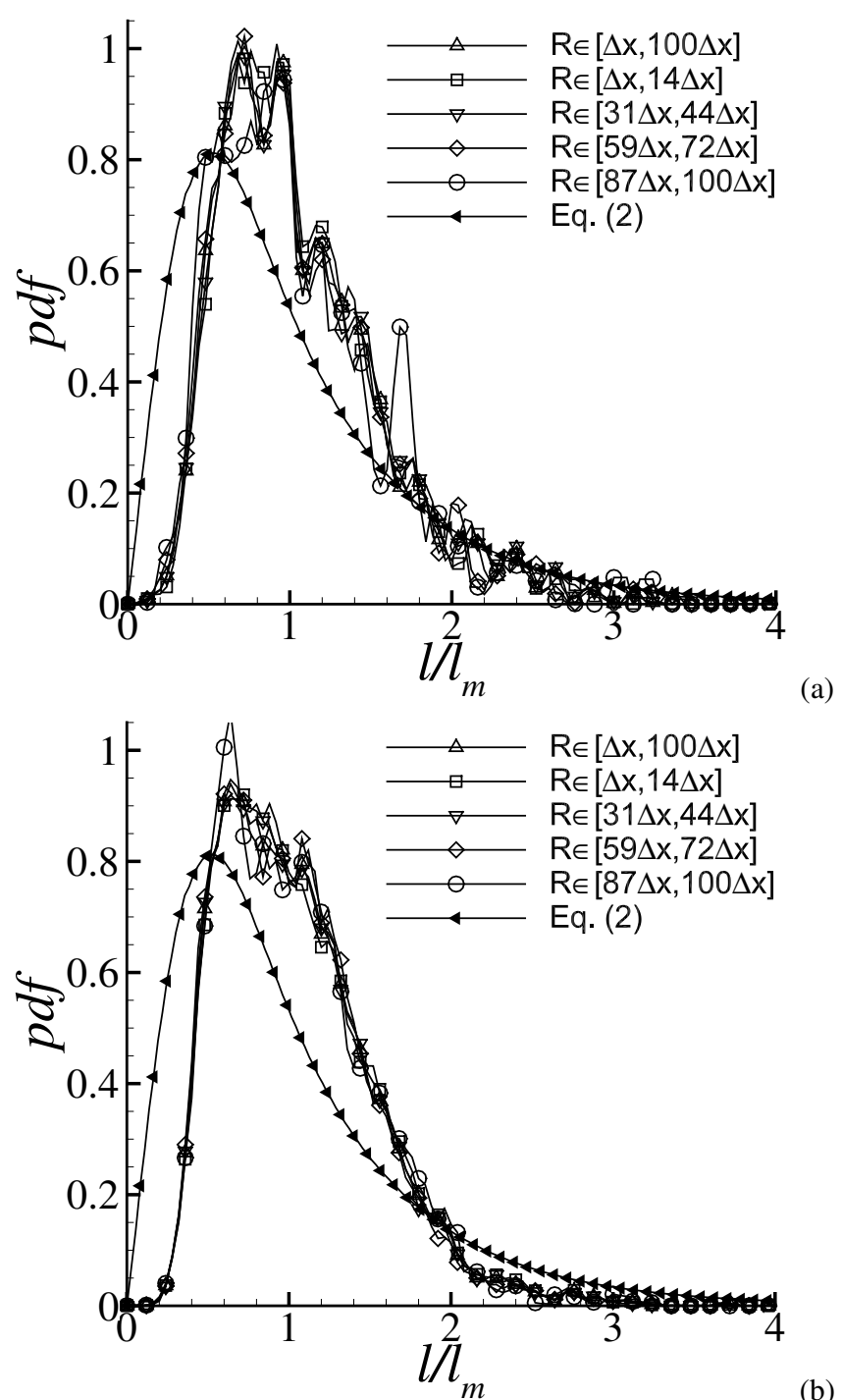

(b)

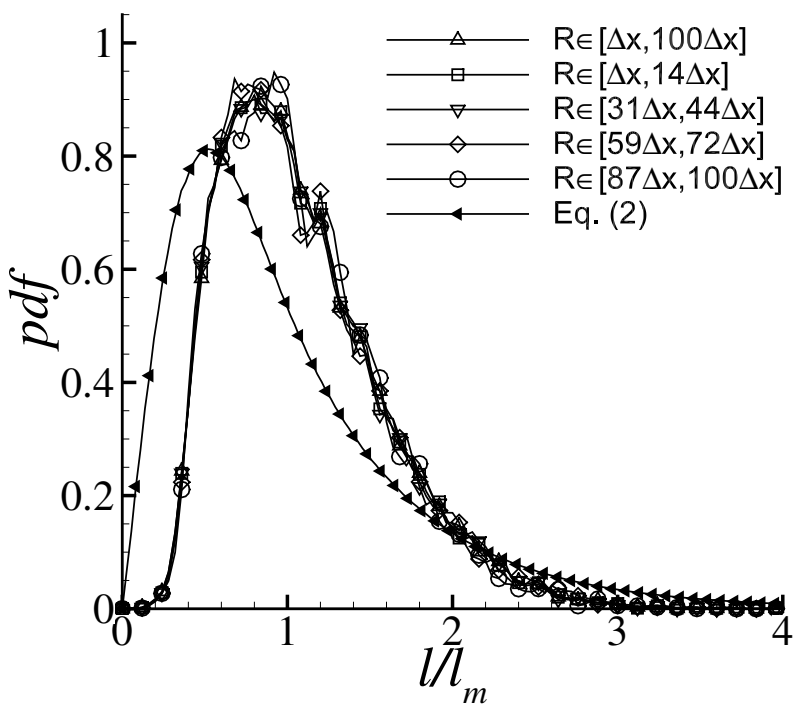

(c)

FIG. 5. The marginal PDFs of the normalized length $\tilde{l}$ for DEs in different ranges of $R$ for case (a) A, (b) B and (c) C, compared with the solution from Eq. (2). 
with the numerical observation from Fig. 6 at the logarithmic scale.
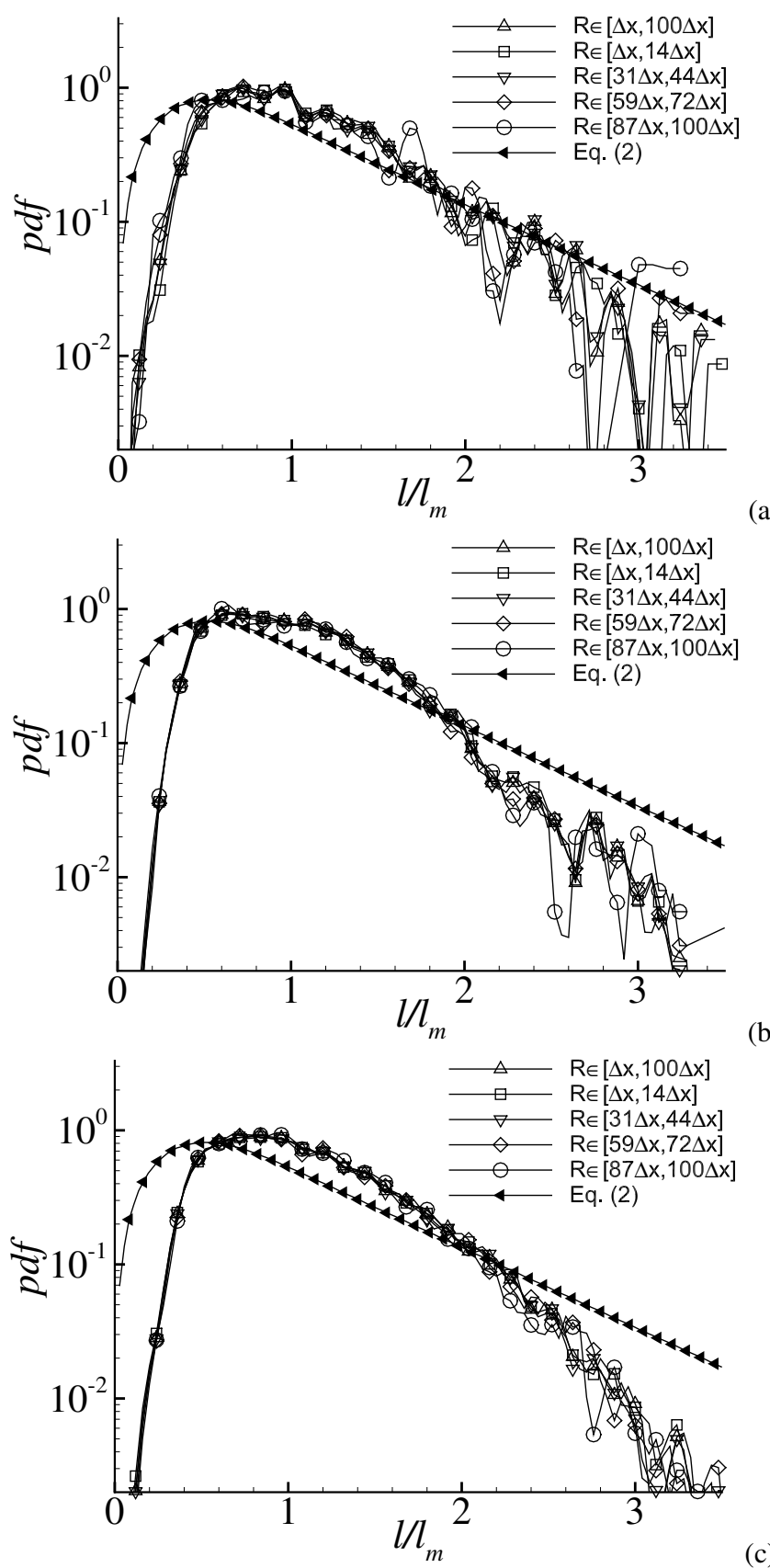

(b)

FIG. 6. Same as Fig. 5, but in the logarithmic scale.

Figure 7 shows $\langle\Delta \phi \mid \tilde{l}\rangle$, the conditional mean of $\Delta \phi$ on the normalized length scale $\tilde{l}$, for the cases considered here. As in Fig. 5, results in different $R$ brackets are presented as well. For each case, all the conditional means with respect to the DE size $\tilde{l}$ have a consistent scaling exponent $K$ for each case, which is interpreted from the fact that turbulence preserves structure self-similarity at multiple scales ${ }^{25}$ and such similarity can be inherited in the structure of DEs (at different $R$ levels). The results shown here also suggest that the structure self-similarity at multiple scales can still be observed in spite

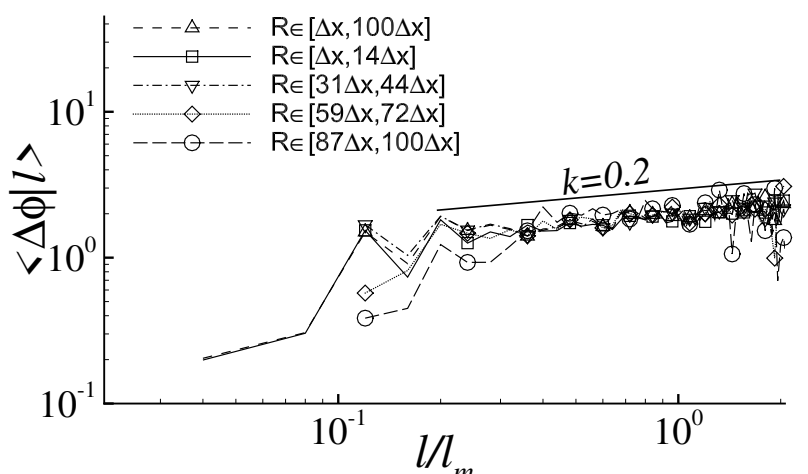

(a)

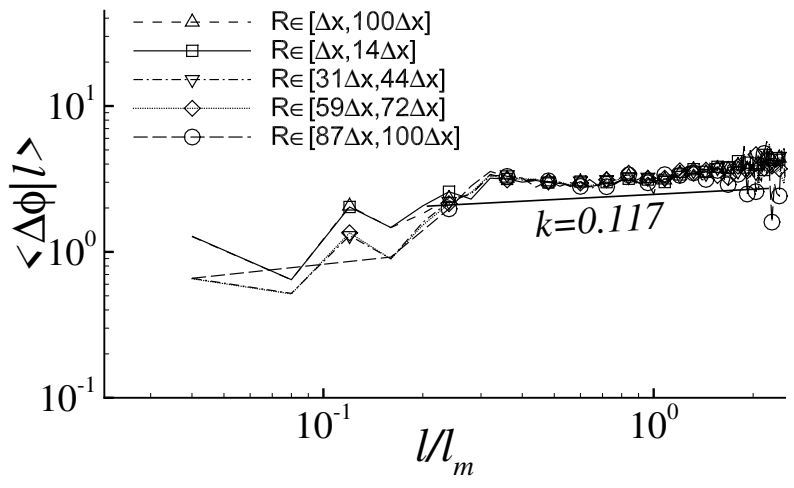

(b)

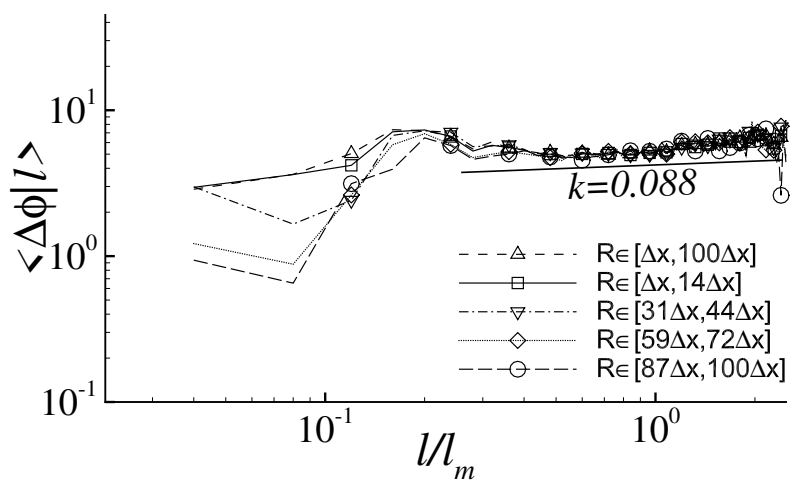

(c)

FIG. 7. Conditional means of $\Delta \phi$ with respect to $\tilde{l}$ for case (a) A, (b) $\mathrm{B}$ and (c) C.

of moderate values of turbulent Reynolds number (this is the case for the DNS considered here), if the structure is appropriately defined. However, the values of the scaling exponent $K$ are found to be significantly different for different cases. Specifically, $K$ decreases with increasing extent of flame wrinkling. Qualitatively, the turbulent premixed flame has both the organized and the random parts. The scaling slope weakens when the noise associated with small scale turbulence part becomes increasingly important. The more intense the turbulence is, the more the flame is corrugated with a stronger influence from the structure randomness, which explains qualitatively the variation of $K$ from case A to case $\mathrm{C}$.

Uniquely, different from other structure identification approaches, the present multi-level DE analysis addresses the non-locality and multi-scale nature of the flame structure in a quantitative manner. On the basis that DEs are space-filling at any scale level, these quantitative results, including the length 
scale PDF in Fig. 5 and the conditional means of the curvature difference in Fig. 7, can then be utilized to reconstruct the topology statistics of the entire flame surface ${ }^{7,8}$, rather than pointwise description.

\section{APPLICATION IN MODEL DEVELOPMENT}

The space-filling feature and non-arbitrariness of DE at different levels make it possible to utilise the DE statistics for the purpose of informing the model development, in the sense that the entire flow can be reconstructed from those of decomposed elements ${ }^{8}$. Specifically if the topological features of the decomposed units can be described by a set of characteristic parameters $\left(p_{1}, p_{2}, \ldots\right)$, once the joint probability density function (PDF) of $p_{i}, P\left(p_{1}, p_{2}, \ldots\right)$ has been modeled, the ensemble average of a statistical property $X$ can then be determined as

$$
\langle X\rangle=\int \ldots \int X\left(p_{1}, \ldots, p_{n}\right) P\left(p_{1}, \ldots, p_{n}\right) d p_{1} \ldots d p_{n} .
$$

Equation (3) can be implemented if and only if the decomposed elements are space-filling, else the results will be biased. In the multi-level DE context, the relation $X\left(p_{1}, \ldots, p_{n}\right)$ can probably be well determined based on the DE structure at different scale levels, which then provides a different approach to express statistical moments.

For instance, the flame curvature is a key parameter in determining the flame surface density and the flame dynamics. The governing equation (i.e. G-equation) of the level-set approach is given by ${ }^{1}$ :

$$
\rho \frac{\partial G}{\partial t}+\rho \vec{u} \cdot \nabla G=\rho S_{d}|\nabla G|,
$$

where $S_{d}$ is the displacement speed which also affects the flame surface area evolution in the following manner 26,27 through its correlation with flame curvature ${ }^{1,24,26,27}$ :

$$
\frac{1}{A} \frac{d A}{d t}=\left(a_{T}+2 S_{d} \kappa\right)
$$

which demonstrates that the flame stretch $\frac{1}{A} \frac{d A}{d t}$ is determined by flame surface tangential strain rate $a_{T}$ and the curvature stretch $2 S_{d} \kappa^{26,27}$. The displacement speed $S_{d}$ can be split in the following manner ${ }^{1,17-20}$

$$
S_{d}=\left(S_{r}+S_{n}\right)-2 D \kappa
$$

where $S_{r}$ and $S_{n}$ denote the contribution from the normal diffusion and reaction to $S_{d}$. Equation (6) implies that the statistics of $\kappa^{2}$ plays a key role in determining the curvature stretch, i.e. $2 S_{d} \kappa=2\left(S_{r}+S_{n}\right) \kappa-4 D \kappa^{2}$, and as for the evolution of the flame surface area ${ }^{26,27}$. The curvature stretch contribution also appears in the FSD transport term in the form of the FSD curvature term ${ }^{26,27}$, i.e. $\overline{\left(2 S_{d} \kappa\right)}{ }_{s} \Sigma_{g e n}=$ $\overline{\left(2\left(S_{r}+S_{n}\right) \kappa\right)}{ }_{s} \Sigma_{g e n}-4 \overline{\left(D \kappa^{2}\right)}{ }_{s} \Sigma_{g e n}$, where $\left.\overline{(Q)}\right)_{s}=\overline{Q|\nabla c|} / \Sigma_{g e n}$ and $\Sigma_{\text {gen }}=\overline{|\nabla c|}$ are the surface-averaging operation of a general quantity $Q$ and the generalised FSD, respectively with the overline indicating Reynolds averaging/LES filtering operation, as appropriate ${ }^{21,22}$. It has been demonstrated elsewhere $^{2,23,28}$ that $-4 \overline{\left(D \kappa^{2}\right)} \Sigma_{s}$ gen plays a key role in determining the behaviour of the FSD curvature term $\overline{\left(2 S_{d} \kappa\right)}{ }_{s} \Sigma_{g e n}$ in the thin reaction zones regime flames. Thus, the statistical behaviours of mean and root-mean-square of $\kappa$ are important from the point of view of turbulent premixed flame modelling.

The unbiased statistics of $\kappa$ can be obtained from the conditional statistics with respect to the multi-level DEs. In simplicity, consider the length $l$ of DE as the only involved parameter in the set $\left(p_{1}, p_{2}, \ldots, p_{n}\right)$ (i.e. $\left.n=1\right)$. If $X$ used here is the fluctuation of $\kappa$ of the decomposed DEs from the flame front, the conditional mean of $X$, i.e. $\kappa_{r m s}$, on $l$ can then be extracted. At different length brackets, the dependence of $\kappa_{r m s}$ and $\kappa_{\text {mean }}$ with respect to $l$ are shown in Fig. 8 .

First of all, the (area-weighted) mean of $\kappa$ is much smaller than $\kappa_{r m s}$ for statistically planar flames, which implies that overall the flame surface does not have preference of bending towards either the burned or unburned side. It can be seen that for different turbulence intensities, at different $R$ levels, $\kappa_{r m s}$ has almost identical dependence on the normalized length scale $\tilde{l}$, even quantitatively. In all cases, $\kappa_{r m s}$ decreases with the increase of the normalized DE length $\tilde{l}$. With the increase of $R, \kappa_{r m s}$ on average becomes larger. The quantity $\kappa_{r m s}$ provides the measure of the disturbance of turbulence on the flame front. Physically, for both velocity and the scalar quantities, turbulent disturbance originates from the random motion of eddies of different sizes. On average, smaller size eddies leads to higher energy dissipation rate, which is determined by the local velocity derivatives. Similarly for the flame front, more rapid change of the local velocity leads to strong corrugation of the flame front and thus larger $\kappa_{r m s}$, while the influence from large scale convection is relatively weak. It is worth mentioning that the results in Fig. 8 are almost independent of the scale $R$ level when the DE length scale is normalized with the respective mean, which makes such statistics more meaningful for the purpose of the development of the models for the quantities which are related to the flame curvature (e.g. sub-grid curvature term in the context of FSD closure).

In the present analysis, the multi-level DE analysis has been conducted where the flame curvature is the scalar in question. The present analysis focuses primarily on non-local curvature statistics that is rarely addressed in the existing literature and reveals its self-similarity at multiple scales even for moderate values of turbulent Reynolds number. This implies that the model equation (i.e. Eq. (2)) for the normalised length scale of the dissipation element $\tilde{l}$ can be used for different length scale windows. This enables one to employ Eq. (3) to understand the non-local flame topology effects on the curvature stretch term $2 S_{d} \kappa$ where both $\kappa_{\text {mean }}$ and $\kappa_{r m s}$ play important roles (e.g. especially $\kappa_{r m s}$ is closely related to $-4 D \kappa^{2}$ contribution of $\left.2 S_{d} \kappa\right)^{19,20}$. This implies that once the displacement speed $S_{d}$ is parameterised in terms of curvature and the joint PDF $P\left(p_{1}, p_{2}, \ldots, p_{n}\right)$ is modelled, Eq. (3) can be utilised to model the rms value of curvature (or curvature variance) in the sub-grid level. This can in turn be exploited for the purpose of modelling of $\overline{2 S_{d} \kappa|\nabla c|}$ in the transport equations of the generalized FSD $\Sigma_{g e n}=\overline{|\nabla c|}$. Interested readers are referred to 

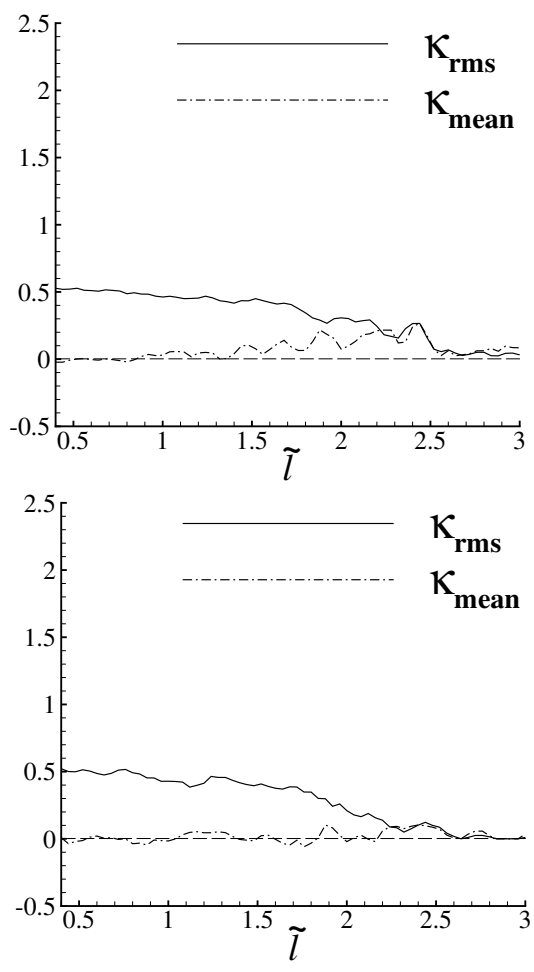

(a)
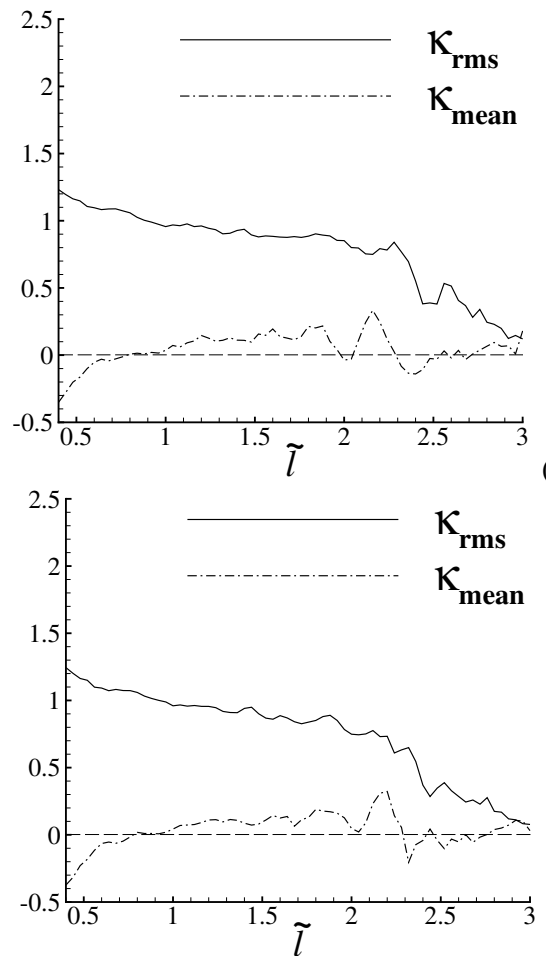

(d)

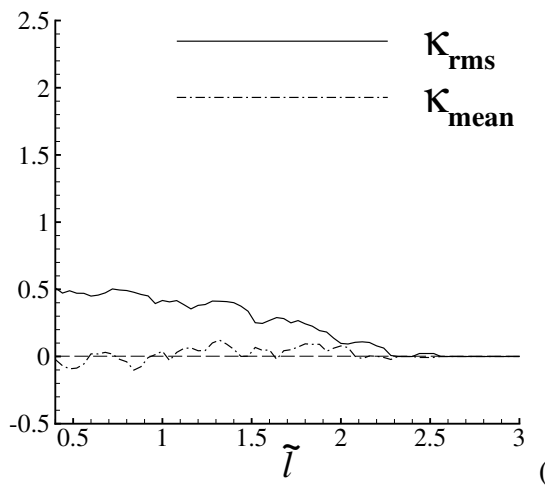

)

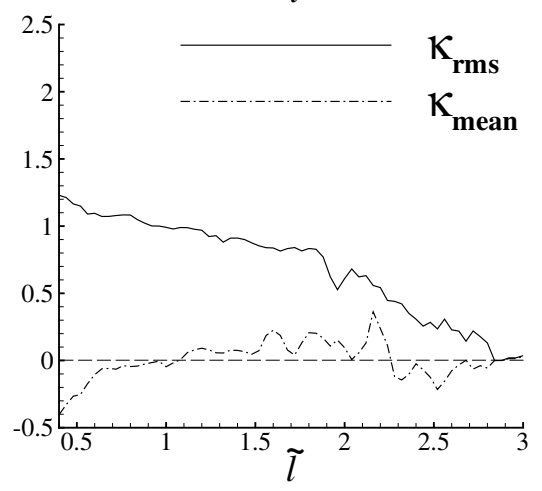

(b)

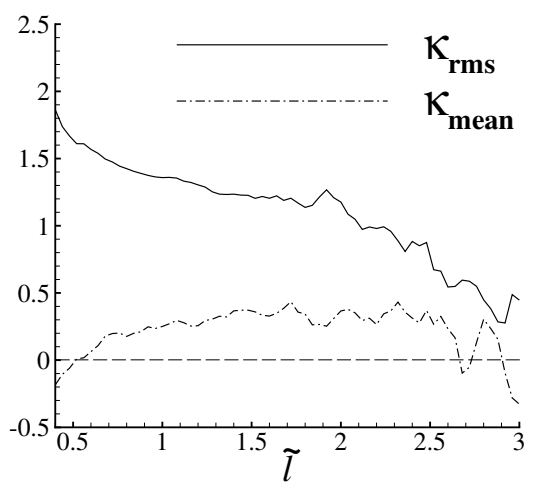

(c)

(e)

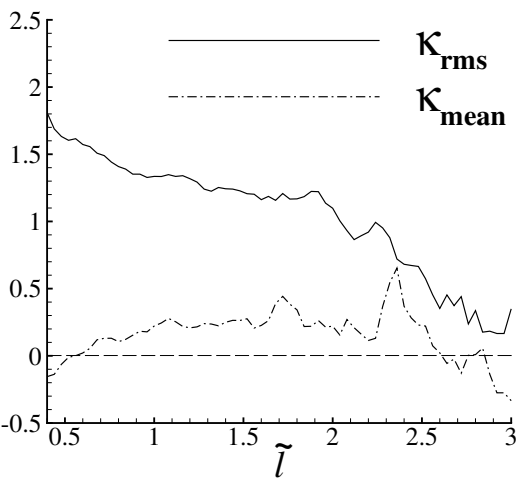

(f)

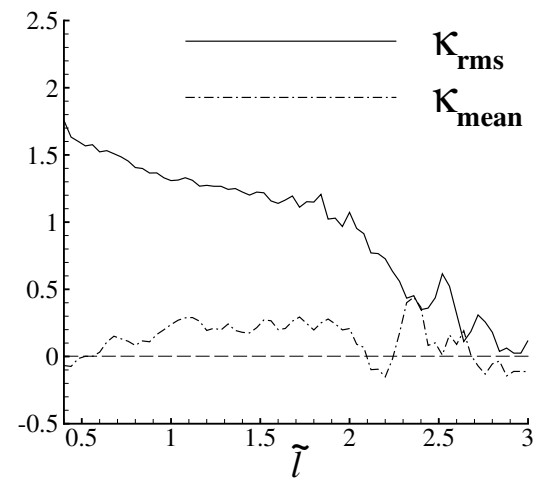

FIG. 8. Dependence of the mean and the fluctuation of $\kappa$ with respect to $l$ for $u^{\prime} / S_{L}=2.5$ (left column), $u^{\prime} / S_{L}=5.0$ (middle column) and $u^{\prime} / S_{L}=7.5$ (right column) at different $R$ levels: (a)-(c): $R=0^{+}$; (d)-(f): $R=40 \Delta x$; (g)-(i): $R=80 \Delta x$.

Herbert et al. ${ }^{29}$ for further discussion on the parameterisation of $S_{d}$ with respect to $\kappa$ for this DNS database, and the parameterisation of $P\left(p_{1}, p_{2}, \ldots, p_{n}\right)$ is kept beyond the scope of this analysis and needs further investigation.

\section{CONCLUSIONS}

In addition to the local statistics of the premixed turbulent flames, it is important to consider the non-local features of the flame surface as a field phenomena. The current analysis focuses on the statistical features of the non-local flame front structure based on the dissipation element (DE) identification at multi-scale levels. Starting from a spatial point on the flame front, the gradient trajectory of the flame curvature reaches a local maximum curvature point and a local minimum curvature point along the ascending and descending directions, re- spectively. A dissipation element is the patch consisting of the points whose gradient trajectories share the same pair of extremal points. The definition of such space-filling elements makes it possible to produce the entire field statistics from those of the decomposed elements. By extending the extremal points at different scale levels $R$, the multi-level DE structure can also be defined for a better understanding of the turbulent flame structure in the sense of scale hierarchy. In this sense, the present multi-level DE is a fundamentally important extension of the DE method, which enables an novel and more general approach to analyze non-local statistics of flame and other complex systems.

Results show that the turbulence intensity determines the flame corrugation and thus the number of DEs, while the probability density functions (PDF) of the normalized length scale $\tilde{l}$ remain unchanged, which is indicative of the structure similarity of turbulence in spite of the moderate values of turbu- 
lent Reynolds number. The number of DEs decreases with increasing $R$. The conditional mean of the curvature difference $\langle\Delta \phi \mid \tilde{l}\rangle$ with respect to the scale $R$ is strongly dependent on the influence of turbulence on the flame. For each case under consideration and each $R$ bracket, there exists a consistent scaling relation between $\langle\Delta \phi \mid \tilde{l}\rangle$ and $\tilde{l}$, which may result also from the turbulence structure similarity. However, the scaling slope decreases with increasing extent of flame wrinkling under high turbulence intensities. Moreover, the present multi-level DE structure is also uniquely insightful for the purpose of model construction in the future. Since DEs at any $R$ level are space-filling, the statistics of the non-local features of the entire flame surface can be constructed from the statistics of the decomposed DEs, as indicated by Eq. (3). This property has been utilised to analyse the mean and rms fluctuation of flame curvature which have relevance in both level-set and FSD based modelling of turbulent premixed flames.

\section{ACKNOWLEDGMENTS}

UA and NC are grateful to EPSRC (EP/R029369/1, EP/P022286/1) and ARCHER for financial and computational support.

Data Availability: The data that support the findings of this study are available from the corresponding author upon reasonable request.

\section{REFERENCES}

${ }^{1}$ N. Peters, Turbulent Combustion, Cambridge University Press, Cambridge, (2000).

${ }^{2}$ N. Chakraborty, R. S. Cant, Direct numerical simulation analysis of the flame surface density transport equation in the context of Large Eddy simulation. Proc. Combust. Inst. 32 (2009), 1445-1453.

${ }^{3}$ V.A. Sabelnikov, A. L. Lipatnikov, S. Nishiki, T. Hasegawa, Application of conditioned structure functions to exploring influence of premixed combustion on two-point turbulence statistics, Proc. Combust. Inst., 37 (2019), 2433-2441.

${ }^{4}$ V.A. Sabelnikov, A. L. Lipatnikov, S. Nishiki, T. Hasegawa, Conditioned structure function method for studying influence of combustion-induced thermal expansion on two-point turbulence statistics in premixed flames, J. Fluid Mech. 867, (2019), 45-76.

${ }^{5}$ P. Brearley, U. Ahmed, N. Chakraborty, A.N. Lipatnikov, "Statistical behaviours of conditioned two-point second-order structure functions in turbulent premixed flames in different combustion regimes" ,

$6,31(2019), 115109$.
${ }^{7}$ L. Wang, N. Peters, The length-scale distribution function of the distance between extremal points in passive scalar turbulence, J. Fluid Mech. 554 (2006), 457-475.

${ }^{8} \mathrm{~L}$. Wang, N. Peters, A new view of flow topology and conditional statistics in turbulence, Phil. Trans. R. Soc. A 2013371 (2012).

${ }^{9}$ L. Wang, N. Peters, Length-scale distribution functions and conditional means for various fields in turbulence, J. Fluid Mech. 608 (2008), 113-138.

${ }^{10}$ L. P. Wang, Y. X. Huang, Multi-level segment analysis: definition and application in turbulent systems, J. Stat. Mech. 06018 (2015).

${ }^{11} \mathrm{~K}$. Jenkins, R. Cant, Direct numerical simulation of turbulent flame kernels, In Knight, D.,and Sakell, L. (Eds.) Recent advances in DNS and LES: Proceedings of the second AFOSR conference, Rutgers - The state University of New Jersey, New Brunswich, USA. Dordrecht, Kluwer, 191-202 (1999).

${ }^{12}$ A. A. Wray, Minimal storage time advancement schemes for spectral methods (Tech. Rep.). Report No. MS 202 A-1, NASA Ames Research Center, California, USA. doi: 10.1099/00221287-136-2-327 (1990).

${ }^{13} \mathrm{U}$. Ahmed, N. Chakraborty, M. Klein, Insights into the Bending Effect in Premixed Turbulent Combustion Using the Flame Surface Density Transport, Combust. Sci. Technol. 191 (2019), NOS. 5-6, 898-920.

${ }^{14} \mathrm{U}$. Ahmed, N. Chakraborty, M. Klein, On the stress-strain alignment in premixed turbulent fames, Sci. Rep. (2019) 9:5092.

${ }^{15}$ T. Poinsot, S. K. Lele, Boundary conditions for direct simulation of compressible viscous flows, J. Comp. Phys. 101 (1992), 104-129.

${ }^{16} \mathrm{M}$. Klein, N. Chakraborty, and S. Ketterl, A comparison of strategies for Direct Numerical Simulation of turbulence chemistry interaction in generic planar turbulent premixed flames. Flow, Turbul. Combust. 99 (2017), 955971.

${ }^{17}$ T. Echekki, J. H. Chen, Analysis of the contribution of curvature to premixed flame propagation. Combust. Flame 118 (1999), 308-311.

${ }^{18} \mathrm{~N}$. Chakraborty, S. Cant, Unsteady effects of strain rate and curvature on turbulent premixed flames in an inflow-outflow configuration. Combust. Flame 137, 129-147 (2004).

${ }^{19} \mathrm{~N}$. Chakraborty, Comparison of displacement speed statistics of turbulent premixed flames in the regimes representing combustion in corrugated flamelets and thin reaction zones, Phys. Fluids 19(10), 105109 (2007).

${ }^{20}$ N. Peters, P. Terhoeven, P., J. H. Chen, T. Echekki, Statistics of Flame Displacement Speeds from Computations of 2-D Unsteady Methane-Air Flames, Proc. of Combustion Institute 27,833-839 (1998).

${ }^{21} \mathrm{~A}$. Trouvé, T. Poinsot, The evolution equation for flame surface density in turbulent premixed combustion, J. Fluid Mech., 278, 1-31 (1994).

${ }^{22}$ M. Boger, D. Veynante, H. Boughanem, A. Trouvé, Direct Numerical Simulation analysis of flame surface density concept for Large Eddy Simulation of turbulent premixed combustion, Proc. of Combustion Institute, 27, $917-$ 925 (1998).

${ }^{23}$ N. Chakraborty, M. Klein, R. S. Cant, Stretch rate effects on displacement speed in turbulent premixed flame kernels in the thin reaction zones regime, Proceedings of the Combustion Institute 31, 1385-1392 (2007).

${ }^{24}$ M. Matalon, B. J. Matkowsky, Flames as gasdynamic discontinuities, $J$. Fluid Mech. 124 (1982), 239-259.

${ }^{25} \mathrm{~A}$. Tsinboer, The essence of turbulence as a physical phenomenon, 2nd edition, Springer Nature Switzerland AG (2019).

${ }^{26} \mathrm{~S}$. Pope, The evolution of surfaces in turbulence, Int. J. Engng Sci. 26(5), 445-469 (1988)

${ }^{27}$ S. M. Candel, T. J. Poinsot, Flame stretch and the balance equation for the flame area, Combust. Sci. Technol., 70(1990), 1-15.

${ }^{28}$ E.R. Hawkes, J.H. Chen, Proc. Combust. Inst. 30, 647-655 (2005).

${ }^{29}$ A. Herbert, U. Ahmed, N. Chakraborty, M. Klein, Applicability of extrapolation relations for curvature and stretch rate dependences of displacement speed for statistically planar turbulent premixed flames, Combustion Theory and Modelling (2020). DOI: 10.1080/13647830.2020.1802066 